\title{
Biological Characterization of an Improved Pyrrole-Based Colchicine Site Agent Identified through Structure-Based Design $\$$
}

\author{
Cristina C. Rohena, Nakul S. Telang, Chenxiao Da, April L. Risinger, James A. Sikorski, \\ Glen E. Kellogg, John T. Gupton, and Susan L. Mooberry \\ Department of Pharmacology and Cancer Therapy \& Research Center, University of Texas Health Science Center at San \\ Antonio, San Antonio, Texas (C.C.R., A.L.R., S.L.M.); Department of Chemistry University of Richmond, Richmond Virginia \\ (N.T., J.T.G.); Department of Medicinal Chemistry and Institute of Structural Biology and Drug Discovery, Virginia Commonwealth \\ University, Richmond, Virginia (C.D., G.E.K.); and Medicinal Chemistry \& Drug Discovery, Chesterfield, Missouri (J.A.S.).
}

Received August 1, 2015; accepted December 9, 2015

\begin{abstract}
A refined model of the colchicine site on tubulin was used to design an improved analog of the pyrrole parent compound, JG03-14. The optimized compound, NT-7-16, was evaluated in biological assays that confirm that it has potent activities as a new colchicine site microtubule depolymerizer. NT-7-16 exhibits antiproliferative and cytotoxic activities against multiple cancer cell lines, with $\mathrm{IC}_{50}$ values of $10-16 \mathrm{nM}$, and it is able to overcome drug resistance mediated by the expression of $\mathrm{P}$-glycoprotein and the $\beta \mathrm{III}$ isotype of tubulin. NT-7-16 initiated the concentration-dependent loss of cellular microtubules and caused the formation of abnormal mitotic spindles, leading to mitotic accumulation. The direct interaction of NT-7-16 with purified tubulin was confirmed, and it was more potent than
\end{abstract}

combretastatin A-4 in these assays. Binding studies verified that NT-7-16 binds to tubulin within the colchicine site. The antitumor effects of NT-7-16 were evaluated in an MDA-MB-435 xenograft model and it had excellent activity at concentrations that were not toxic. A second compound, NT-9-21, which contains dichloro moieties in place of the 3,5-dibromo substituents of NT7-16, had a poorer fit within the colchicine site as predicted by modeling and the Hydropathic INTeractions score. Biological evaluations showed that NT-9-21 has 10-fold lower potency than NT-7-16, confirming the modeling predictions. These studies highlight the value of the refined colchicine-site model and identify a new pyrrole-based colchicine-site agent with potent in vitro activities and promising in vivo antitumor actions.

\section{Introduction}

Microtubules are dynamic structures that play critical roles in intracellular transport, protein trafficking, and cell division. The ability to disrupt these processes has proven useful for anticancer therapy, and microtubule disrupting drugs continue to be a mainstay in the treatment of a wide variety of adult and pediatric cancers (Jordan and Wilson, 2004; Dumontet and Jordan, 2010). Chemically diverse microtubule targeting agents (MTAs) were initially derived from a variety of natural products, including paclitaxel and vinblastine, but advances in synthetic chemistry have led to the development of new microtubule targeting drugs via semi-synthesis or total synthesis, including docetaxel, cabazitaxel, vinorelbine, and eribulin. New MTAs with improved clinical efficacy and

We gratefully acknowledge the National Institutes of Health [Grant R15 CA67236-05A1] for support of the synthetic chemistry portion of this project. Funding for the biological evaluations was provided by the President's Council Research Excellence Award and Greehey Distinguished Chair in Molecular Targeted Therapies to S.L.M

dx.doi.org/10.1124/mol.115.101592.

S This article has supplemental material available at molpharm. aspetjournals.org. different spectrums of activity, including the ability to overcome drug resistance mechanisms, continue to advance into clinical use.

To date, five distinct binding sites for MTAs on tubulin/ microtubules have been identified: two for microtubule stabilizers and three for microtubule depolymerizers. The microtubule stabilizer sites are the taxoid site on $\beta$-tubulin in the interior of the microtubule (Nogales et al., 1995; Xiao et al., 2006) and the laulimalide/peloruside site that is also located on $\beta$-tubulin, but on the exterior of the microtubule (Huzil et al., 2008; Bennett et al., 2010). Drug occupancy within these sites stimulates tubulin polymer formation, leading to a higher density of cellular microtubules. In contrast, microtubule depolymerizers inhibit tubulin polymerization and cause a loss of cellular microtubules. Three microtubule destabilizer binding sites have been identified: the vinca domain (Hamel, 2002), the maytansine site (Prota et al., 2014), and the colchicine site (Hamel, 2003). Vinblastine binds within a deep pocket formed between two adjacent $\alpha \beta$ tubulin heterodimers; occupancy within this site disrupts both the longitudinal and lateral interactions between tubulin heterodimers (Gigant et al., 2005). Multiple clinically useful MTAs bind 
within the vinca domain. The maytansine site is close to, but not overlapping with, the vinca domain, and binding prevents the formation of longitudinal associations of microtubule protofilaments (Prota et al., 2014). Maytansine analogs have recently found utility as antibody-drug conjugates (Verma et al., 2012). The colchicine site consists of a deep pocket in $\beta$-tubulin at the $\alpha \beta$ tubulin interface (Ravelli et al., 2004; Dorléans et al., 2009). Colchicine prevents microtubule elongation and destabilizes protofilament interactions, leading to microtubule depolymerization. Whereas colchicine was the first MTA to be identified, it was found to be too toxic for the treatment of cancer, yet it showed clinical utility in the treatment of gout and Mediterranean familial fever. Some less toxic colchicine site compounds that have been evaluated clinically include 2-methoxyestradiol, the combretastatins CA-4P and CA-1P, ABT-751, and NPI-2358, and newergeneration colchicine site agents continue to advance to clinical trials with the hope that improved anticancer drugs can be identified ( $\mathrm{Lu}$ et al., 2012). One of the most important attributes of colchicine site agents is their ability to circumvent multiple modes of resistance to clinically approved MTAs, including expression of the P-glycoprotein (Pgp) drug efflux pump or the $\beta$ III isotype of tubulin. The discovery and development of novel compounds that bind in a distinct manner within the colchicine binding site are important to more fully exploit the clinical potential of this site in hopes of identifying a therapeutic lead compound with excellent efficacy and low toxicity.

Compounds with a wide range of structural diversity can interact within the colchicine site (Lu et al., 2012). X-ray crystallography has defined multiple binding modes within the site, and these differences in binding modes might underlie some of the differences in activities among colchicine site agents (Dorléans et al., 2009). Analogous to the vinca domain, the different binding poses prompted the suggestion that the colchicine site be referred to as the colchicine domain, taking into account these various orientations (Dorléans et al., 2009). A continuing challenge in the rational development of new colchicine site agents is the low resolution $(\sim 3.6 \AA)$ of the existing crystal structures of this site, and a further challenge in designing new colchicine site agents with improved binding properties is the inherent flexibility of the colchicine binding pocket as predicted by molecular simulations (Ravelli et al., 2004; Dorléans et al., 2009; Chakraborti et al., 2012) and the fact that more than 56 chemical scaffolds can interact within this pocket.

Over the past 5 years, our goal has been to iteratively refine the model of the colchicine site to allow more informed design of selective, potent compounds that might overcome the limitations of earlier generation colchicine site agents (Tripathi et al., 2008; Da et al., 2012, 2013a,b). Combining an ensemble docking approach (applying five crystal structures of $\alpha \beta$-tubulin) with the biologic activities of 59 compounds allowed us to identify optimal substituents for favorable hydrophobic interactions and hydrogen-bonding opportunities. Consequently, a near-atomic-resolution molecular model of the colchicine binding site was revealed that possesses three major binding pockets (Da et al., 2013a). This information allowed us to design an optimized analog of the previously described pyrrole-based colchicine site agent, JG-03-14 (Mooberry et al., 2007). The new analog, NT-7-16, is a potent microtubule depolymerizing agent that overcomes multiple drug resistance mechanisms and has excellent antitumor effects with no evident toxicities. These results validate our docking model for the rational design of improved pyrrole-based colchicine site agents.

\section{Materials and Methods}

Materials. Paclitaxel and combretastatin A-4 (CA-4) were purchased from Sigma-Aldrich (St. Louis, MO). All compounds were solubilized in dimethylsulfoxide (DMSO; Sigma-Aldrich).

Modeling. Molecular modeling procedures are generally as reported previously (Da et al., 2013a). Briefly, X-ray crystal structure models of $\alpha \beta$-tubulin (pdbids: 1SA0, 1SA1, 3HKC, 3HKD, and 3HKE) (Ravelli et al., 2004; Dorléans et al., 2009) were obtained from the RCSB protein data bank. We used Sybyl 8.1 (Tripos, LP, St. Louis, MO) to prepare protein and small molecule models for docking and the Tripos force field with Gasteiger-Hückel charges for model optimization. Ligands were docked with GOLD 5.1 (Jones et al., 1995) by generating 100 conformations for each compound that were initially analyzed by GoldScore and further by rescoring with HINT (Kellogg and Abraham, 2000; Sarkar and Kellogg, 2010). HINT is a scoring algorithm developed to enumerate and evaluate hydrophobic as well as polar interactions (e.g., Coulombic, hydrogen bonding) that is based on the experimental measurements of small molecule $\log \mathrm{P}_{\text {octanol/water }}$. $\log P$ is the free energy for solute transfer between the two solvents. In previous studies, HINT scores have been shown to correlate with $\Delta \Delta \mathrm{G}$ such that $\sim 500$ HINT score units $=1 \mathrm{kcal} \mathrm{mol}^{-1}$ (Burnett et al., 2001; Cozzini et al., 2004). The "active" conformation was selected from the models at all five colchicine site structures as the conformation with both a high HINT score and high similarity to the conformation of the complexed ligand in these structures. It should be noted that the crystallographic models for the bound ligands are only approximate because of the low resolution of the protein structures.

Chemical Synthesis of NT-7-16 and NT-9-21. Detailed methods for the synthesis and structure determination of these new chemical entities are provided in the Supplemental Material. All purified reaction products gave thin-layer chromatography results, flash chromatograms, and proton and carbon nuclear magnetic resonance spectra consistent with a single, homogeneous substance with purity exceeding $95 \%$.

Cell Lines. The A-10, HeLa, and SK-OV-3 cell lines were purchased directly from the American Type Culture Collection (Manassas, VA). MDA-MB-435 cells were obtained from the Lombardi Cancer Center of Georgetown University (Washington, D.C.) and validated by American Type Culture Collection. The HeLa wild-type $\beta$ III (WT $\beta$ III) and SK-OV-3/MDR-1-6/6 cell lines were described previously (Risinger et al., 2008). MDA-MB-435 cells were maintained in improved minimum essential medium (Richter's Modification; Gibco, Life Technologies, Grand Island, NY) supplemented with $10 \%$ fetal bovine serum, $25 \mu \mathrm{g} / \mathrm{ml}$ gentamicin in a humidified $37^{\circ} \mathrm{C}$ incubator with $5 \% \mathrm{CO}_{2}$. The wild-type $\beta \mathrm{III}$ cell line was grown and maintained in Dulbecco's modified Eagle's medium (Gibco, Life Technologies) with $10 \%$ fetal bovine serum, $50 \mu \mathrm{g} / \mathrm{ml}$ gentamicin in a humidified $37^{\circ} \mathrm{C}$ incubator with $5 \% \mathrm{CO}_{2}$. The other cell lines were maintained in basal medium Eagle's (Sigma-Aldrich) supplemented with $10 \%$ fetal bovine serum and $50 \mu \mathrm{g} / \mathrm{ml}$ gentamicin in a humidified $37^{\circ} \mathrm{C}$ incubator with $5 \% \mathrm{CO}_{2}$. Cell stocks were stored in liquid nitrogen, and all experiments performed within six months of retrieval.

Fluorescence Microscopy. A-10 and HeLa cells were plated on glass coverslips and allowed to attach for 24 hours. Cells were treated with vehicle (DMSO), CA-4, NT-7-16, or NT-9-21 at specified concentrations for 18 hours. Microtubules were visualized with a $\beta$-tubulin antibody (Clone Tub2.1, Sigma-Aldrich), the DNA stained with DAPI (Sigma-Aldrich), and images acquired with a Nikon Eclipse Ti80 microscope with the Nikon Advanced Research Imaging Software (Tokyo, Japan). To calculate the $\mathrm{EC}_{50}$ for microtubule depolymerization, the 
percent microtubule loss in A-10 cells was estimated microscopically over a range of concentrations as previously described (Gangjee et al., 2010; Lee et al., 2010). All experiments were performed a minimum of three times.

Inhibition of Cellular Proliferation. The sulforhodamine B (SRB) assay was used to measure the antiproliferative and cytotoxic effects of the compounds as previously described. Briefly, cells were plated in 96-well plates and allowed to attach for 24 hours. Cells were then treated with a range of drug concentrations for 48 hours, fixed, and protein stained with $\mathrm{SRB}$ dye. $\mathrm{IC}_{50}$ values were calculated from the linear portions of the log-dose response curves of three independent experiments, each conducted in triplicate. Values are expressed as mean \pm S.D.

Cell Cycle Analysis. HeLa cells were treated for 18 hours with NT-7-16, NT-9-21, vehicle (DMSO), or paclitaxel, and the drug's effects on cell cycle progression were evaluated by flow cytometry as previously described (Gangjee et al., 2010; Lee et al., 2010). Cells were harvested and stained with Krishan's reagent and analyzed with a BD Biosciences BD LSRII flow cytometer (BD Biosciences, Franklin Lakes, NJ).

Tubulin Polymerization. The effects of the compounds on purified porcine brain tubulin polymerization (Cytoskeleton, Denver, CO) were monitored at $340 \mathrm{~nm}$ with a SpectraMax plate reader. The assay mixture contained $2 \mathrm{mg} / \mathrm{ml}$ tubulin in GPEM buffer (80 $\mathrm{mM}$ PIPES, pH 6.8; $1 \mathrm{mM} \mathrm{MgCl}_{2}$; and $1 \mathrm{mM}$ EGTA) containing $1 \mathrm{mM}$ GTP and $10 \%$ glycerol and DMSO as vehicle $(0.5 \% \mathrm{v} / \mathrm{v})$ or specified drug in $100 \mu \mathrm{l}$ reactions at $37^{\circ} \mathrm{C}$.

Electron Microscopy. Aliquots from tubulin polymerization experiments as described here were collected after 60 minute reaction time and fixed by mixing with equal volumes of $4 \%$ gluteraldehyde solution (Electron Microscopy Sciences, Hatfield, PA). Reaction mixtures were mounted on 200 mesh copper grids, washed with a 10\% cytochrome $\mathrm{C}$ solution (Sigma-Aldrich), and negatively stained with $8 \%$ uranyl acetate. Microtubules were visualized using a JEOL100CX transmission electron microscope with a range of 2000-100,000 × magnification.

Colchicine Displacement. The ability of NT-7-16 to displace colchicine from tubulin was evaluated using a fluorescent colchicine displacement assay (Bhattacharyya and Wolff, 1974). Reaction mixtures containing $2 \mu \mathrm{M}$ tubulin with or without $2 \mu \mathrm{M}$ colchicine were incubated for 2 hours at $37^{\circ} \mathrm{C}$ with vehicle (DMSO), $20 \mu \mathrm{M} \mathrm{CA}-4$, $100 \mu \mathrm{M}$ vinblastine, or a range of concentrations $(2-10 \mu \mathrm{M})$ of NT-7-16. The fluorescence of the samples was analyzed using a Horiba Fluoromax-3 spectrofluorometer (Horiba Jobin Yvon, Edison, NJ) using an excitation wavelength of $380 \mathrm{~nm}$ and an emission wavelength of 438 $\mathrm{nm}$. The fluorescence values were normalized by subtracting the buffer alone and setting the fluorescence of tubulin and colchicine as $100 \%$.

In Vivo Studies. Six-week-old athymic nude $\left(F_{o x n} 1^{n u} / F_{o x n} 1^{n u}\right)$ female mice were obtained from Harlan (Indianapolis, IN) and injected with MDA-MB-435 tumor fragments on each flank. When tumors reached approximately $200 \mathrm{~mm}^{3}$, mice were placed into three groups of five mice each (10 tumors) that were assigned to give the same average tumor size per group and randomly assigned a treatment condition. Mice were injected i.p. with either $20 \mathrm{mg} / \mathrm{kg}$ paclitaxel $2 \times$ weekly for 2 weeks or $75 \mathrm{mg} / \mathrm{kg}$ NT-7-16 daily for 14 days. Tumor volume was measured as width $\times$ length $\times$ height in $\mathrm{mm}^{3}$, and weight gain/loss was monitored. NT-7-16 was prepared in a 2:1:7 solution (Tween 80:DMSO:phosphate-buffered saline) in a total volume of $0.2 \mathrm{ml}$ per injection. Paclitaxel was prepared in 1:1:18 (Cremophor:EtOH:phosphate-buffered saline) in a total volume of $0.2 \mathrm{ml}$ per injection. The studies were conducted in accordance with the National Institutes of Health guidelines as described in the Guide for the Care and Use of Laboratory Animals and approved by the University of Texas Health Sciences Center at San Antonio Institutional Animal Care and Use Committee. The animals were housed in an American Association for Laboratory Animal Care-approved facility, where food and water were provided ad libitum.
Statistical Studies. For all antiprolilferative studies, an average of at least three independent experimental values were used to generate the $\mathrm{IC}_{50}$ values; standard deviation was calculated to illustrate the variability of the data. For in vivo studies, statistical analysis of final tumor volumes was performed using a one-way analysis of variance (ANOVA) with a Tukey's post hoc test. Statistical analysis of animal weight loss on day 14 was performed using a one-way ANOVA with a Dunnet's post hoc test to compare each drugtreated condition with the control. An unpaired $t$ test was used to compare the wet weights of control and NT-7-16 treated tumors at completion of the trial.

\section{Results}

The initial lead pyrrole compound JG-03-14 (Fig. 1A), a tetra-substituted brominated pyrrole, has microtubule depolymerizing activities and antitumor effects (Mooberry et al., 2007). The chemical structure and positioning of JG-03-14 within the colchicine site are shown in Fig. 1B. Studies were initiated to identify the structure activity relationships within this class of pyrrole compounds that lead to high potencies for both inhibition of cancer cell proliferation and microtubuledepolymerizing effects. As part of this optimization process, the HINT scores of the pyrroles were calculated because HINT scores have been shown in a large and diverse number of computational experiments to correlate with the free energy of binding and are useful in comparative studies of drug binding (Spyrakis et al., 2007). Our goal was to design analogs of the parent compound with higher HINT scores to optimize the hydrogen binding within the Cys $241 \beta$ and Ala $354 \beta$ containing sub-pocket of the colchicine site.

The lack of an optimal pose of JG-03-14 within the colchicine site was predicted based on its high $\mathrm{EC}_{50} / \mathrm{IC}_{50}$ ratio. This ratio of the concentration that causes $50 \%$ depolymerization of cellular microtubules $\left(\mathrm{EC}_{50}\right)$ divided by the concentration that causes $50 \%$ inhibition of cancer cell proliferation $\left(\mathrm{IC}_{50}\right)$ has proven useful to compare on-target microtubuledependent effects with off-target cytotoxic actions. The low $\mathrm{EC}_{50} / \mathrm{IC}_{50}$ ratio of 2.3 seen with the colchicine site binding agent CA-4 (Table 1) is indicative of a close correlation between cytotoxicity and microtubule depolymerization, suggesting that interruption of microtubule-dependent activities is largely responsible for its cytotoxic effects. In comparison, higher ratios suggest that off-target effects, independent of microtubule depolymerization, are major contributors to a compound's cytotoxic activities. The high $\mathrm{EC}_{50} / \mathrm{IC}_{50}$ ratio of 13 for JG-03-14 (Table 1) suggests that JG-03-14 has off-target cytotoxic effects in addition to its microtubule depolymerizing activity, which was confirmed (Gupton et al., 2000).

Our goal was to design improved analogs of JG-03-14 based on a large data set of diverse colchicine site-interacting compounds that was used to refine the model of the colchicine site (Da et al., 2013a). Based on molecular simulations and acknowledgment of the importance of hydrophobic interactions within the colchicine site, a 2,3,4 trimethoxyphenyl analog, designated NT-7-16, was identified with an optimal fit (Fig. 1, A and B). The additional methoxy group at the 2-position of the phenyl ring in NT-7-16 provides opportunities for enhanced hydrophobic interactions with Ala354 $\beta$ that are not present in the parent compound (Fig. 1B).

NT-7-16 was synthesized and its biological activities evaluated. A second compound, designated NT-9-21 (Fig. 1A), which 
A<smiles>CCOC(=O)c1[nH]c(Br)c(-c2ccc(OC)c(OC)c2)c1Br</smiles><smiles>CCOC(=O)c1[nH]c(Br)c(-c2ccc(OC)c(OC)c2OC)c1Br</smiles>

NT-7-16<smiles>CCOC(=O)c1[nH]c(Cl)c(-c2ccc(OC)c(OC)c2OC)c1Cl</smiles>

NT-9-21
B

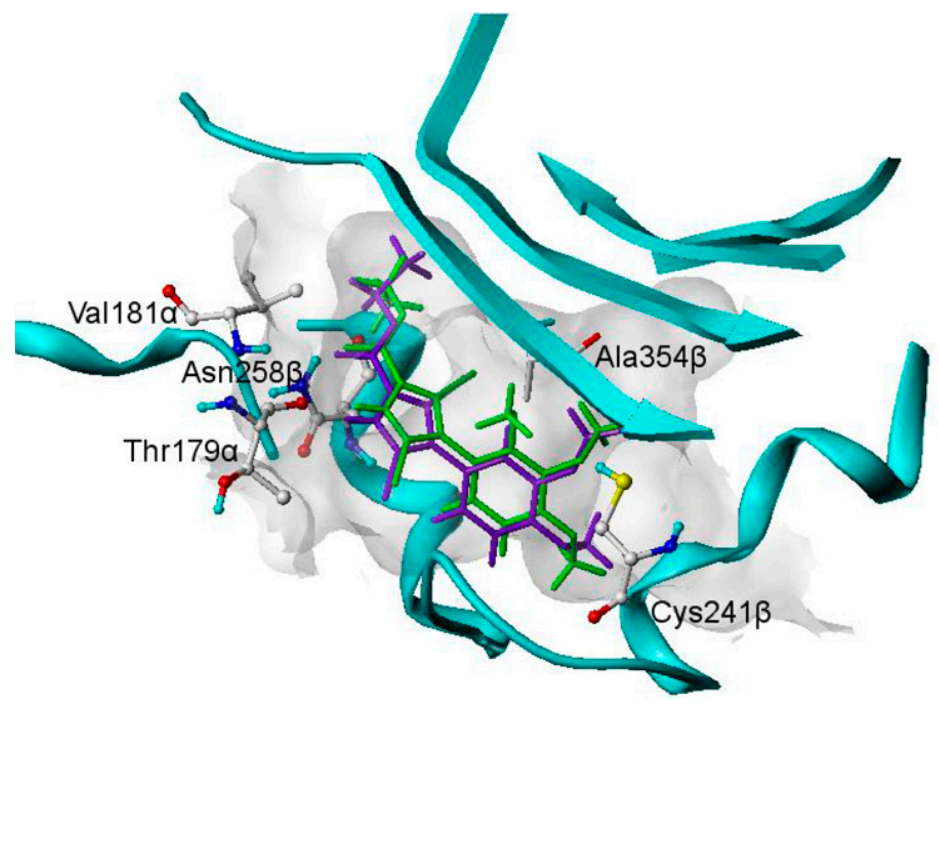

Fig. 1. Chemical structures of JG-03-14, NT-7-16, and NT-9-21 (A). The modeling of JG-03-14 (purple) and NT-7-16 (green) within the colchicine site of tubulin. The methoxy group at the 2-position of the phenyl ring in NT-7-16 has opportunities for enhanced hydrophobic interactions with Ala354 $\beta$ that the parent JG-03-14 does not have.

is identical to NT-7-16 but with dichloro substituents replacing the dibromo groups at C3 and C5, has a lower HINT score (Table 1) and was also synthesized and evaluated as an additional test for the modeling predictions. The biological evaluations showed that NT-7-16 is more potent than the parent compound, with an $\mathrm{IC}_{50}$ for inhibition of proliferation of $10.4 \mathrm{nM}$ in the MDA-MB-435 cell line, a 3.4-fold improvement over JG-03-14 (Table 1). Additionally, as would be expected for a better fit with in the colchicine site, NT-7-16 has an $\mathrm{EC}_{50}$ for loss of cellular microtubules of $37 \mathrm{nM}$, a 13-fold improvement over the parent compound. The $\mathrm{EC}_{50} / \mathrm{IC}_{50}$ ratio of NT-7-16 of 3.6 indicates a close association between the concentration that inhibits cellular proliferation and that causes loss of cellular microtubules, consistent with on-target effects. The potencies and $\mathrm{EC}_{50} / \mathrm{IC}_{50}$ ratio of NT-7-16 are comparable to those obtained with CA-4, a compound that sits in a distinct pocket of the colchicine site and is the active product of the clinically evaluated prodrug, CA-4P. These results show that the HINT score and modeling predictions were accurate in predicting optimal interactions of pyrrole compounds within the colchicine binding pocket, yielding a compound with high biological potencies and a low $\mathrm{EC}_{50} / \mathrm{IC}_{50}$ ratio. The biological evaluations are consistent with NT-7-16 having an improved fit within the colchicine site, consistent with the modeling predictions.

The second pyrrole analog, NT-9-21 (Fig. 1A), has a HINT score of 772, which suggests an improved fit compared with JG-03-14, but not as optimal as NT-7-16 (Table 1). Consistent with a predicted poorer fit within the colchicine site, NT-9-21 had a 27-fold higher EC $_{50}$ value than NT-7-16 and an 11-fold decrease in potency for inhibition of proliferation in the MDAMB-453 cell line (Table 1 ). The $\mathrm{EC}_{50} / \mathrm{IC}_{50}$ ratio of 8.6 for this compound suggests additional cytotoxic mechanisms outside of its ability to interact with the colchicine site. These results confirm the value of the docking site model we have developed for the binding of pyrroles within the colchicine domain, and its quantification with HINT scores can help predict optimal interactions within the refined model and facilitate the design of new pyrrole compounds with much improved activities.

One advantage of many compounds that bind within the colchicine domain is the ability to overcome drug resistance mediated by the expression of the $\beta$ III isotype of tubulin. One difference between $\beta$ III compared with other isotypes is the substitution of Cys241 with a serine in the A-pocket of the colchicine site, which can limit drug binding (Joe et al., 2008). The ability of NT-7-16 to overcome drug resistance mediated by expression of the $\beta$ III isotype of tubulin was evaluated using an isogenic cell line pair of parental and $\beta$ IIIoverexpressing HeLa cells (WT $\beta$ III). $\beta$ III tubulin-mediated drug resistance to paclitaxel was observed in the WT $\beta$ III cells as indicted by a relative resistance $\left(R_{\mathrm{r}}\right)$ value of 8.6 , which is obtained by dividing the $\mathrm{IC}_{50}$ of the $\beta$ III-expressing cell line by

\section{TABLE 1}

Biological potencies and HINT scores

The effects of the compounds on the inhibition of cancer cell proliferation were evaluated using the SRB assay in MDA-MB- 435 cells $\left(n=3 \pm\right.$ S.D.). The $\mathrm{EC}_{50}$ values for microtubule loss in A-10 cells were calculated from concentration response curves for cellular microtubule loss $(n=3 \pm$ S.D.). The HINT scores of the pyrrole compounds were calculated.

\begin{tabular}{lcccc}
\hline \multicolumn{1}{c}{ Activity } & CA-4 & JG-03-14 & NT-7-16 & NT-9-21 \\
\hline $\mathrm{IC}_{50}(\mathrm{nM})$ & $4.4 \pm 0.5$ & $35.5 \pm 0.2$ & $10.4 \pm 0.5$ & $116 \pm 4$ \\
$\mathrm{EC}_{50}(\mathrm{nM})$ & $10 \pm 1$ & $470 \pm 20$ & $37 \pm 2$ & $1000 \pm 100$ \\
$\mathrm{EC}_{50} / \mathrm{IC}$ & 2.3 & 13 & 3.6 & 8.6 \\
HINT score & NA & 697 & 824 & 772 \\
\hline
\end{tabular}


the $\mathrm{IC}_{50}$ of the parental HeLa cell line. In contrast, in this cellline pair, CA-4 has an $R_{r}$ value of 1.0 , showing that CA-4 is able to overcome drug resistance mediated by $\beta$ III tubulin (Table 2). NT-7-16 and NT-9-21, with $\mathrm{R}_{\mathrm{r}}$ values of 1.2 and 1.1, respectively, can also circumvent drug resistance mediated by $\beta$ III tubulin (Table 2).

A second isogenic cell line pair was used to evaluate the ability of the new compounds to circumvent drug resistance mediated by the drug efflux pump Pgp. The SK-OV-3-MDR-1/ M-6-6 line is a Pgp-expressing clone derived from the parental SK-OV-3 cell line. This Pgp-expressing subline is resistant to the effects of paclitaxel as evidenced by an $R_{\mathrm{r}}$ value of 220 (Table 2), but it retains sensitivity to CA-4, with a $R_{\mathrm{r}}$ value of 0.61 (Table 2). Both NT-7-16 and NT-9-21 have the ability to circumvent drug resistance mediated by Pgp as indicated by the low $R_{\mathrm{r}}$ values of 0.70 or 0.67 for each compound, respectively (Table 2). These results demonstrate that cells expressing Pgp remain sensitive to NT-7-16 and NT-9-21, consistent with the effects of CA-4. Thus, in contrast to many clinically useful anticancer drugs, including paclitaxel, the new pyrrole-based colchicine site agents are able to overcome drug resistance mediated by $\beta$ III tubulin or Pgp expression.

The effects of NT-7-16 and NT-9-21 on interphase and mitotic microtubules were evaluated in A-10 and HeLa cells, respectively. Normal microtubule arrays were present in vehicle-treated cells, with the microtubules extending from the microtubule organizing center in the center of the cell toward the periphery (Fig. 2). The positive control, CA-4, caused a loss of microtubules with an $\mathrm{EC}_{50}$ for cellular microtubule depolymerization of $10 \mathrm{nM}$, where loss of microtubules was observed at the cell periphery but were retained in the vicinity of the microtubule organizing center (Fig. 2). Consistent with the effects of CA-4, NT-7-16 and NT-9-21 each caused loss of interphase microtubules. NT-7-16 caused extensive, concentration-dependent microtubule loss between 25 and 50 nM (Fig. 2; Supplemental Fig. 1). NT-9-21 caused similar microtubule loss at concentrations in the low micromolar range (Fig. 2).

The loss of interphase microtubules initiated by either NT7-16 or NT-9-21 was accompanied by a notable increase in the number of cells in mitosis, consistent with interruption of the formation of functional mitotic spindles. The effects of the compounds on mitotic spindle structures were evaluated in HeLa cells. Cells treated with vehicle had normal, bipolar mitotic spindles with the chromosomes aligned at the metaphase plate (Fig. 3A). In contrast, in cells treated with $15 \mathrm{nM}$ NT-7-16, the appearance of lagging chromosomes was evident and was more pronounced at $25 \mathrm{nM}$ (Fig. 3A, right panels). NT-9-21 caused similar effects but at much higher concentrations (data not shown). Consistent with these mitotic spindle defects, an accumulation of cells in $\mathrm{G}_{2} / \mathrm{M}$ was also observed (Fig. 3B). The cell cycle profile of vehicle-treated cells indicates that the majority of cells were in the $G_{1}$ phase of the cell cycle (Fig. 3B). The microtubule stabilizer, paclitaxel, caused the majority of the HeLa cells to accumulate in $\mathrm{G}_{2} / \mathrm{M}$, consistent with mitotic arrest (Fig. 3B). NT-7-16 and NT-9-21 also caused $\mathrm{G}_{2} / \mathrm{M}$ accumulation with NT-7-16 being much more potent, causing pronounced $\mathrm{G}_{2} / \mathrm{M}$ accumulation at $25 \mathrm{nM}$, while $500 \mathrm{nM} \mathrm{NT}-9-21$ was required to cause the same change in cell cycle distribution (Fig. 3B).

Due to its superior potency and better predicted fit within the colchicine site, we focused further studies on NT-7-16. The ability of NT-7-16 to interact directly with porcine brain tubulin was evaluated turbidimetrically. Robust polymerization was observed in vehicle-treated samples while the positive control, CA-4, inhibited this polymerization (Fig. 4A). Consistent with our cellular studies showing depolymerization of interphase microtubules (Fig. 2), NT-7-16 inhibited the polymerization of purified tubulin in a concentrationdependent manner (Fig. 4A). Interestingly, NT-7-16 was more potent than CA-4 in this assay as $5 \mu \mathrm{M}$ NT-7-16 was more effective than $10 \mu \mathrm{M} \mathrm{CA}-4$ for inhibiting microtubule polymerization. Once this direct interaction with tubulin was confirmed, the effects of NT-7-16 and CA-4 on tubulin structures were evaluated by electron microscopy. Consistent with the increased turbidity observed in Fig. 4A, microtubule polymers were observed in samples treated with vehicle while a $10 \mu \mathrm{M}$ concentration of CA-4 reduced the number and length of these microtubule polymers (Fig. 4B). Similarly, very few microtubules were observed in the presence of $2.5 \mu \mathrm{M}$ NT-7-16 and with a $5 \mu \mathrm{M}$ concentration essentially no microtubules were observed by electron microscopy (Fig. 4B). The finding that NT-7-16 was more potent than CA-4 for inhibiting tubulin polymerization is striking, suggesting that NT-7-16 does have optimal interactions with tubulin, while in the cellular assays CA-4 was more potent.

Colchicine displacement assays were performed to evaluate whether NT-7-16 is able to prevent colchicine binding consistent with occupancy of the colchicine site. When bound to tubulin, colchicine is constrained, which produces fluorescence that was defined as $100 \%$ colchicine binding. The effects of NT-7-16, CA-4, and vinblastine on colchicine binding were evaluated (Fig. 4C). At $20 \mu \mathrm{M}$, a concentration stoichiometric to tubulin, the colchicine site binding agent CA-4 decreased colchicine fluorescence by $72 \%$, consistent with occupancy at the colchicine site. In contrast, the negative control, vinblastine, which binds to the distinct vinca domain on tubulin, had little effect even at the super-stoichiometric concentration of

TABLE 2

The ability of compounds to overcome drug resistance mediated by BIII tubulin or P-glycoprotein expression was evaluated using pairs of isogenic cell lines.

The $\mathrm{IC}_{50}$ values were determined in each cell line using the SRB assay and are expressed $(\mathrm{n}=3 \pm$ S.D.). The relative resistance $\left(\mathrm{R}_{\mathrm{r}}\right)$ values were determined by dividing the $\mathrm{IC}_{50}$ of the resistant cell line by the $\mathrm{IC}_{50}$ of the parental cell line. Paclitaxel and CA-4 were used a positive and negative controls, respectively.

\begin{tabular}{lcccccc}
\hline Compound & HeLa & HeLa WT $\beta$ III & $\mathrm{R}_{\mathrm{r}}$ & SK-OV-3 & SK-OV-3-MDR-1/M-6-6 & $\mathrm{R}_{\mathrm{r}}$ \\
\hline & $m M$ & $n M$ & & $n M$ & $n M$ & \\
NT-7-16 & $12.9 \pm 0.8$ & $15.7 \pm 0.2$ & 1.2 & $15.2 \pm 0.8$ & $10.6 \pm 0.3$ & 0.70 \\
NT-9-21 & $131 \pm 8$ & $140 \pm 20$ & 1.1 & $210 \pm 70$ & $140 \pm 80$ & 0.67 \\
CA-4 & $3.3 \pm 0.4$ & $3.3 \pm 0.3$ & 1.0 & $3.3 \pm 0.3$ & $2.0 \pm 0.9$ & 0.61 \\
Paclitaxel & $2.8 \pm 0.4$ & $24 \pm 4$ & 8.6 & $5.0 \pm 0.6$ & $1,120 \pm 60$ & 220 \\
\hline
\end{tabular}



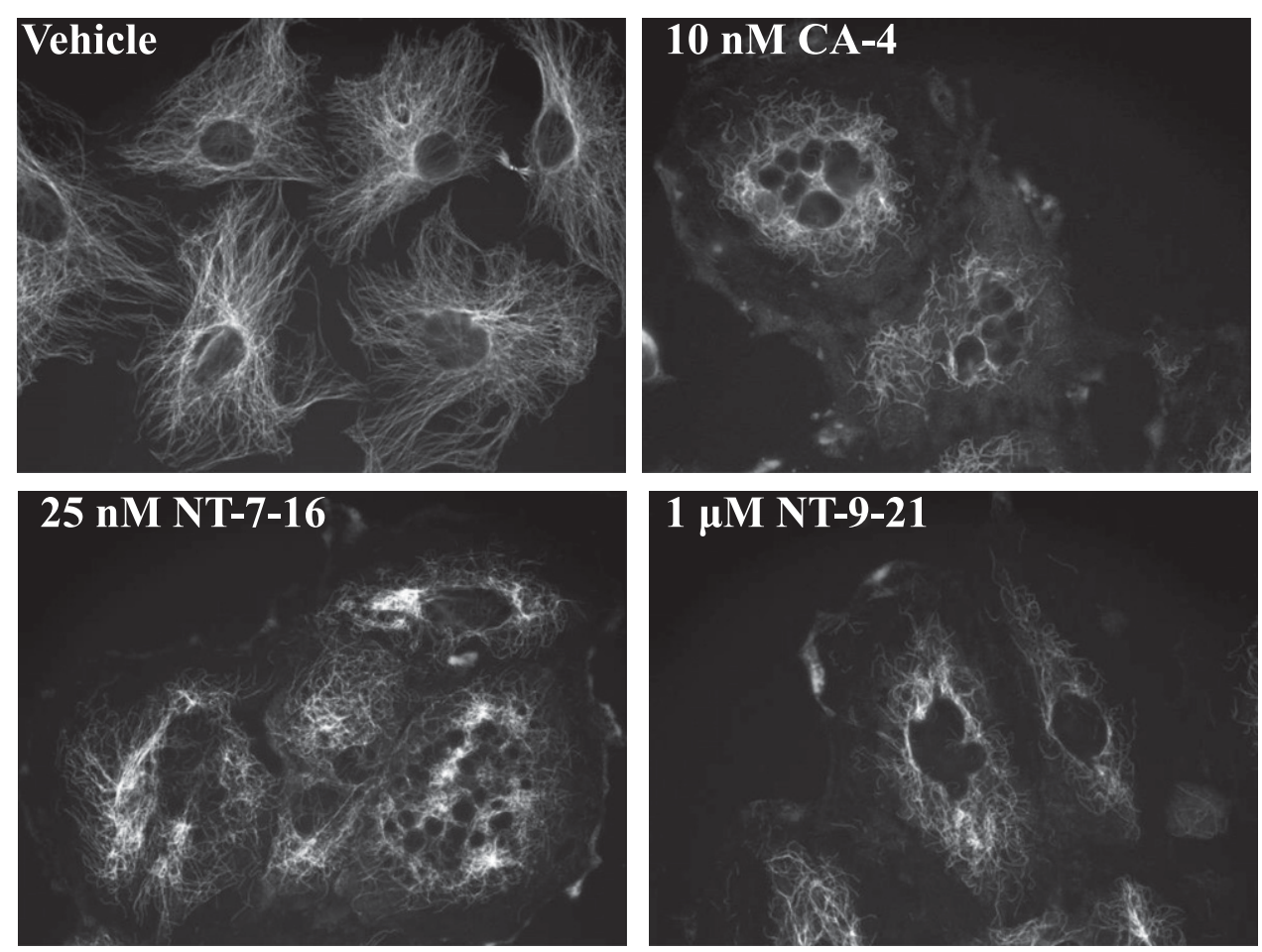

Fig. 2. Effects on interphase microtubules. A-10 cells were treated with vehicle (DMSO), $10 \mathrm{nM} \mathrm{CA-4,} 25 \mathrm{nM}$ NT-7-16, or $1 \mu \mathrm{M}$ NT-9-21 for 18 hours. Microtubules were visualized by indirect immunofluorescence using a $\beta$-tubulin antibody.
$100 \mu \mathrm{M}$ (Fig. 4C). Consistent with occupancy of the colchicine site, NT-7-16 caused a dose-dependent decrease in the fluorescence of tubulin-bound colchicine with a $10 \mu \mathrm{M}$ concentration of NT-7-16 inhibiting this fluorescence by $78 \%$ (Fig. 4C).

The promising potent antiproliferative effects of NT-7-16 against multiple cancer cell lines prompted its evaluations in in vivo models of cancer to evaluate its potency, efficacy and toxicity. The antitumor effects of NT-7-16 were evaluated in a MDA-MB-435 human xenograft model in athymic nude female mice. Initial dose tolerance tests were conducted to identify an optimal dose and schedule. A maximal tolerated dose was not identified in these trials and $75 \mathrm{mg} / \mathrm{kg}$ was found to be the highest dose possible based on the limited aqueous solubility of NT-7-16. The in vivo trial was initiated when the tumors had an average size of $200 \mathrm{~mm}^{3}$. A $75 \mathrm{mg} / \mathrm{kg}$ dose of NT-7-16 was administered i.p. daily over a period of 14 days for a total dose of $1,050 \mathrm{mg} / \mathrm{kg}$. The positive control, paclitaxel, was dosed twice a week i.p. at $20 \mathrm{mg} / \mathrm{kg}$ for a total dose of 80 $\mathrm{mg} / \mathrm{kg}$. The results of this trial show that NT-7-16 had antitumor effects that were significantly different from control tumors $(P=0.0018)$ whereas paclitaxel had no significant effect compared with control or NT-7-16 treated tumors at these doses and schedules (Fig. 5, A and B). When the individual tumor volumes were graphed at the conclusion of the trial, it was interesting that the tumors in the NT-7-16 treatment group were much smaller with less variability in tumor size as compared with either the paclitaxel or control groups (Fig. 5B). At day 14, the average tumor burden for NT$7-16$ treated mice was $521 \mathrm{~mm}^{3}$ (range $197-858 \mathrm{~mm}^{3}$ ), whereas the average tumor volume for the control and paclitaxel groups were 1,182 (range 564-1,871 $\mathrm{mm}^{3}$ ), and $833 \mathrm{~mm}^{3}$ (range 237-1,694 $\mathrm{mm}^{3}$ ), respectively. Additionally, when the wet weights of the tumors were evaluated at the conclusion of the trial, the animals treated with NT-7-16 had an average tumor weight of $381 \mathrm{mg}$ whereas the control animals had an average tumor weight of $899 \mathrm{mg}$, further demonstrating that NT-7-16 significantly inhibited tumor growth $(P=0.0007)$ (Supplemental Fig. 2). These tumor measurements highlight the excellent antitumor effects of NT-7-16. Daily dosing of NT$7-16$ at $75 \mathrm{mg} / \mathrm{kg}$ did not lead to any evidence of overt toxicity and no notable change in the weight of the animals was measured as compared with the controls (Fig. 5C). In comparison, significant cumulative weight loss compared with control mice $(P=0.0014)$ was observed in the paclitaxeltreated group at day 14 with no significant inhibition of tumor size.

In conclusion, our data show that NT-7-16 is a potent microtubule depolymerizer that binds to the colchicine site and possesses significant antitumor effects with no evidence of toxicity. The modeling predictions and the activities of NT-716 demonstrate the value of our refined model for the design of improved agents that bind within the pyrrole sub-pocket of the colchicine site.

\section{Discussion}

This study presents the biological validations of the rational synthesis of pyrrole-containing colchicine site agents based on our refined colchicine binding site model, docking and 3D QSAR methodologies. With this refined binding model, we designed molecules that, as predicted, bind more optimally within the colchicine domain and have superior biological potencies and specificity as compared with earlier generation pyrrole-based colchicine site agents. In particular, the 2,3,4 trimethoxyphenyl analog, NT-7-16, showed excellent, low nanomolar potency in a variety of sensitive and drug resistant cancer cell lines, was more potent than CA- 4 in its ability to inhibit the polymerization purified tubulin, and had excellent antitumor activity in a MDA-MB-435 xenograft model without evidence of toxicities. 

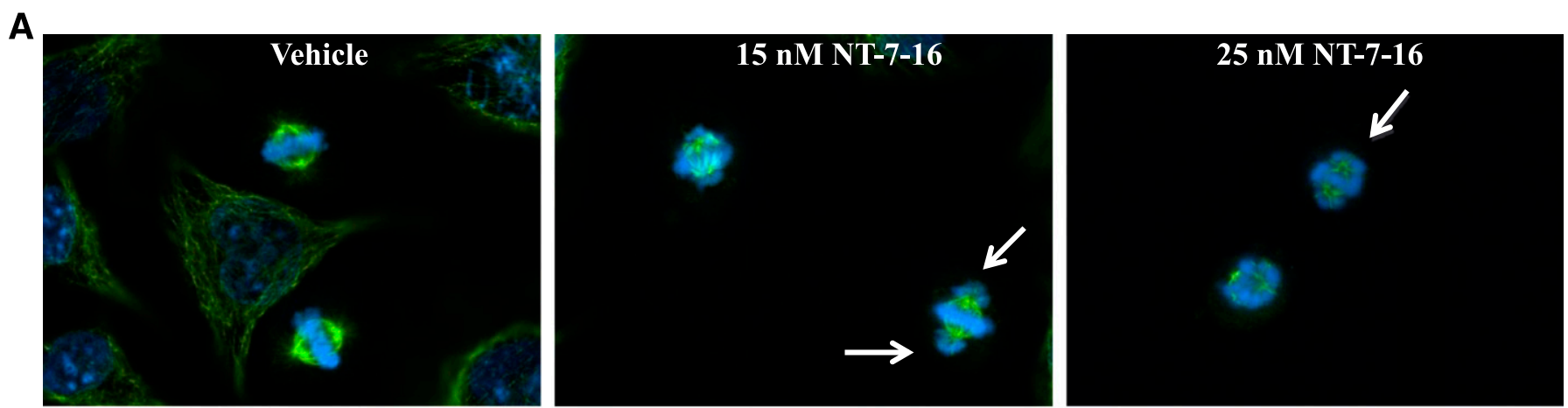

\section{B}
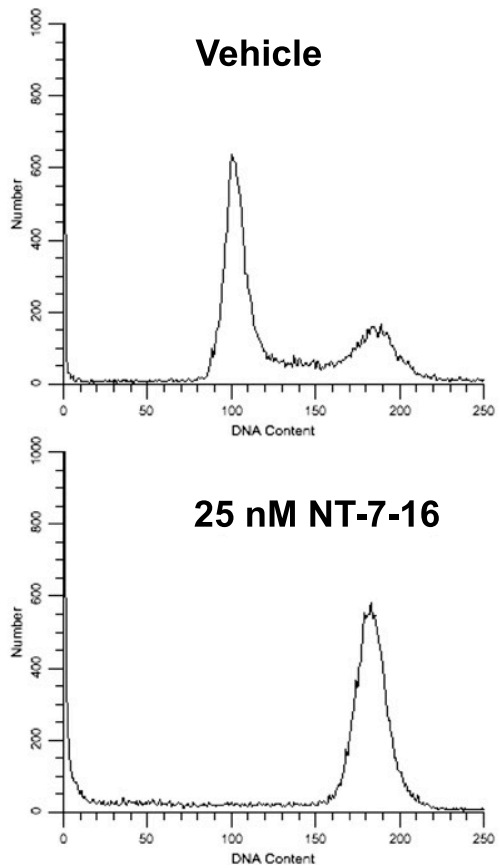
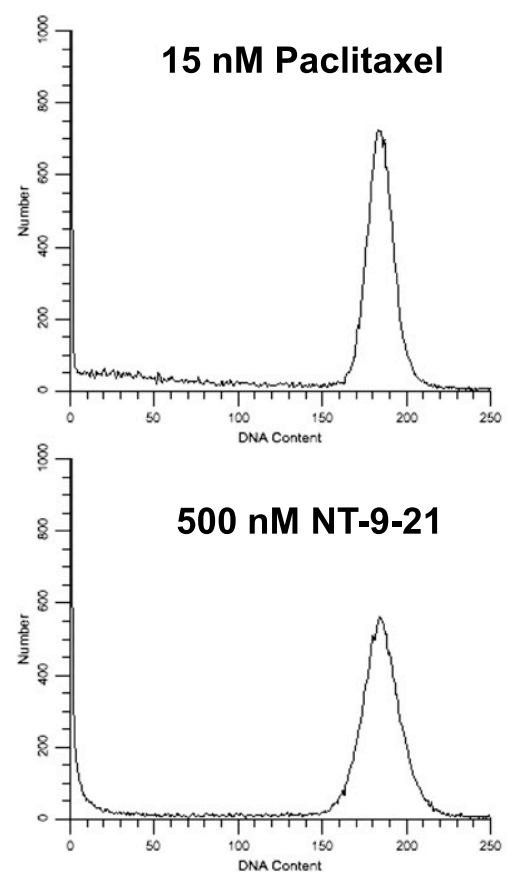

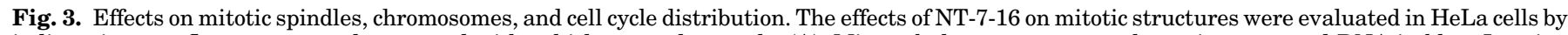

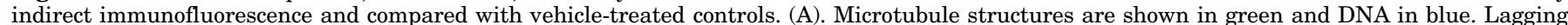

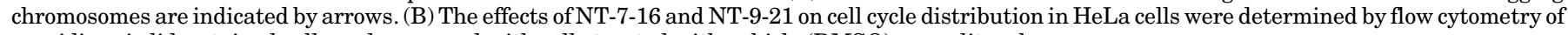
propidium iodide-stained cells and compared with cells treated with vehicle (DMSO) or paclitaxel.

Our previous modeling and biological studies showed that an ethyl ester is optimal at the $\mathrm{C} 2$ substitution of the pyrrole, providing favorable alkyl length and hydropathic properties into the site's C2 pocket, named based on the $2^{\text {nd }}$ ring of colchicine (Da et al., 2012). Shortening or lengthening the C2 ethyl ester group or adding more polar C2 amide functionality diminished optimal interactions and decreased potency and selectivity. We previously evaluated pyrrole C4 phenyl substituents and their interaction within the A pocket of the colchicine site. These modeling simulations and biological evaluation of 18 compounds with various phenyl substitutions at $\mathrm{C} 4$ identified the importance of hydrogen bonding between the compound and Cys241 $\beta$, which is in an otherwise hydrophobic pocket (Da et al., 2013b). The refined model shows that the precise placement of hydrogen bond acceptors and hydrophobes in this pocket is critical: the 3-methoxyphenyl substituent at $\mathrm{C} 4$ has a five-fold higher $\mathrm{EC}_{50}$, and the 4-methoxyphenyl substituent has a 14-fold higher $\mathrm{EC}_{50}$ than the 3,4-dimethoxyphenyl substituent found in JG-03-14 (Da et al., 2013a).
Furthermore, the potency provided by the 2,3,4-trimethoxyphenyl group at C4 in NT-7-16 stands in sharp contrast to the dramatically lower activity reported for the corresponding 3,4,5-trimethoxy-phenyl analog (Da et al., 2013a). Although maximizing the engagement of pocket hydrophobic residues was seemingly desirable, placing a 3,4,5-trimethoxyphenyl at C4 yielded a compound with a 145-fold higher $\mathrm{EC}_{50}$ compared with JG-03-14, presumably due to clashes of the ring's 5 -position methoxy with the pocket wall. Interestingly, one of the key differences between the $\beta$ III isotype of tubulin and other isotypes is the replacement of the A-pocket Cys241 $\beta$ with a serine, which modeling indicates provides a key hydrogen-bonding interaction with the most active pyrrole compounds, particularly those with a low $\mathrm{EC}_{50} / \mathrm{IC}_{50}$ ratio. The $-\mathrm{OH}$ group of serine would be expected to be a stronger hydrogen bond donor to the 2-methoxy group than the-SH of cysteine, yielding tighter interactions between compounds with appropriately-placed acceptors and this residue, such as NT-7-16. Overall, such compounds would be expected to interact more strongly with the $\beta$ III isotype of tubulin, which is implicated in multidrug resistance to other classes of 

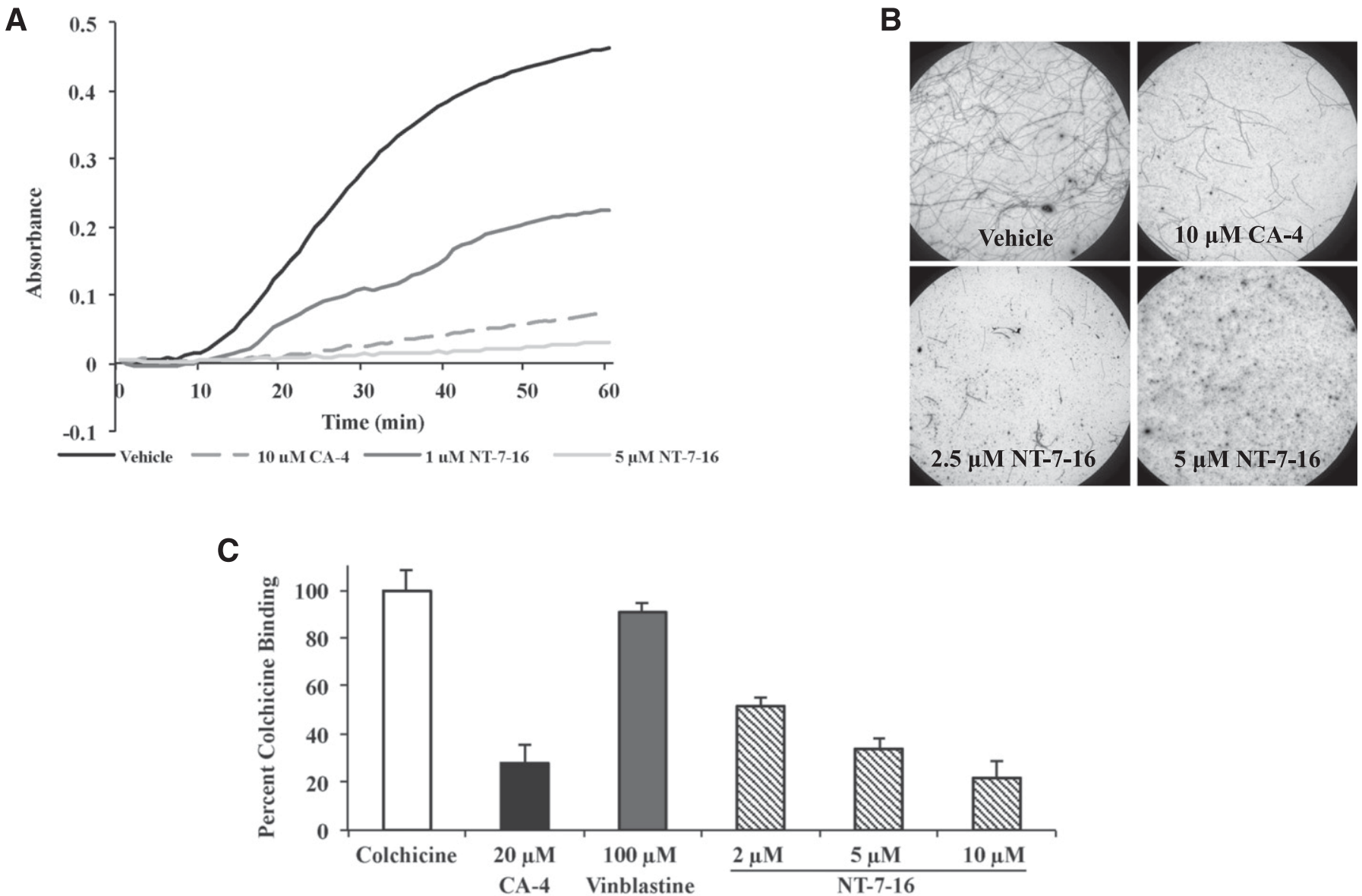

Fig. 4. Effects on tubulin polymerization and colchicine displacement. The ability of NT-7-16 to interact directly with tubulin was evaluated. (A) The polymerization of purified porcine brain tubulin was monitored by absorbance at $340 \mathrm{~nm}$ after incubation with vehicle (DMSO), $10 \mu \mathrm{M}$ CA-4, or NT-7-16 at 1 or $5 \mu \mathrm{M}$. (B) The effects of NT-7-16 on microtubule structures were evaluated by electron microscopy. Samples were treated similarly as in (A) and microtubules visualized by electron microscopy. Shown are representative images at $2000 \times$ magnification of tubulin in the presence of vehicle, $10 \mu \mathrm{M} C A$ 4 , or NT-7-16 at 2.5 or $5 \mu \mathrm{M}$. (C) The effects of NT-7-16 on colchicine binding were evaluated fluorometrically. CA-4 was used as a positive control and vinblastine as a negative control. Data are presented as an average of three experiments \pm S.D.

microtubule targeted agents. This is indeed what is seen with these compounds in that they show no loss in potency or efficacy in HeLa cells overexpressing the $\beta$ III isotype.

Our molecular model also suggested opportunities for further optimization of the pyrroles. First, while optimizing substituents for the $\mathrm{C} 2$ position of the pyrrole, we observed that alkyl groups longer than butyl led to a substantive decrease in potency, which we rationalized as a second binding mode where the C2 group was splayed in the pocket entrance as it became too large for the pocket. In the higher-activity binding mode, the pocket entrance is occupied by the pyrrole C5 substituents, Br (NT-7-16) and Cl (NT-9-21), neither of which appear to take full advantage of the properties of residues in the entrance of the pocket. Second, the C3 position of pyrrole has been explored only cursorily. The 3,5-dibromo substituted NT-7-16 outperformed the 3,5-dichloro substituted NT-9-21 in terms of both efficacy and potency. Bromine is: a) larger than $\mathrm{Cl}, \mathrm{b}$ ) more hydrophobic than $\mathrm{Cl}$, and c) a C-Br bond is longer than a $\mathrm{C}-\mathrm{Cl}$ bond, therefore our models predict that NT-7-16 has stronger hydrophobic interactions within the pocket than NT-9-21. This is further is corroborated by our biologic data. The model indicates that the site, which is really more of a corner, provides a relatively small volume and is notably hydrophobic. One lesson learned from our earlier optimization of the $\mathrm{C} 2$ pocket (Da et al., 2012) was that very minor structural differences that compromise tight hydrophobic associations can have large effects on $\mathrm{EC}_{50}$ : the ethyl to methyl ester substitution resulted in a 17-fold increase in $\mathrm{EC}_{50}$. In that context, the differences between $\mathrm{Br}$ and $\mathrm{Cl}$ at $\mathrm{C} 3$ are not surprising; the $\mathrm{Br}$ of NT-7-16 similarly should make tighter contacts with the pocket than the $\mathrm{Cl}$ of NT-9-21. Nonetheless, the 27-fold difference in $\mathrm{EC}_{50}$ between the NT-9-21 dichloro and the NT-7-16 dibromo compounds suggests that some future optimization of that position may yield unanticipated results.

The colchicine domain is well known for the wide range of chemical structures and poses that can occupy this largely unstructured site. The orientations of colchicine, ABT-751 and podophyllotoxin were used to describe the "main site" by Dorléans and colleagues (Dorléans et al., 2009). However, even within this "main site" there are major differences in binding poses and interactions with tubulin ABT-751 overlaps extensively with colchicine, but is buried further into $\beta$-tubulin, which facilitates interactions with Tyr $202 \beta$ but eliminates the interaction with the $\alpha$ subunit. Dorléans et al. (2009) additionally describe a deeper pocket buried in $\beta$-tubulin that 


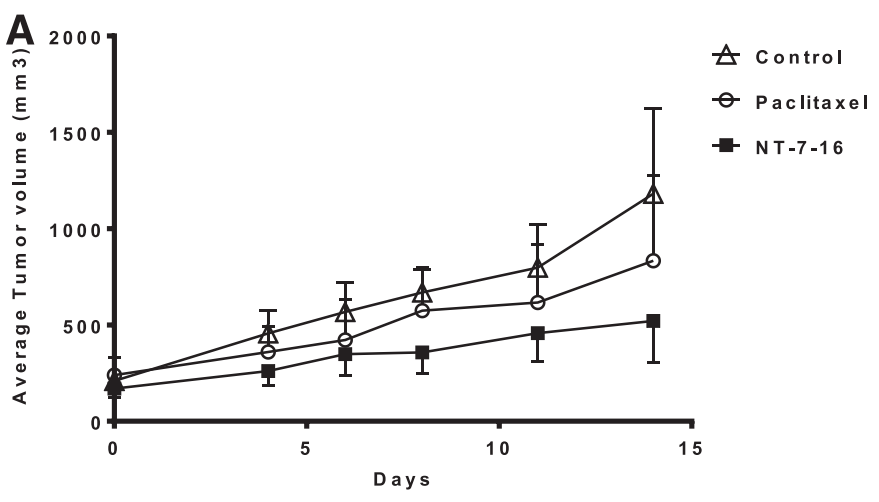

B

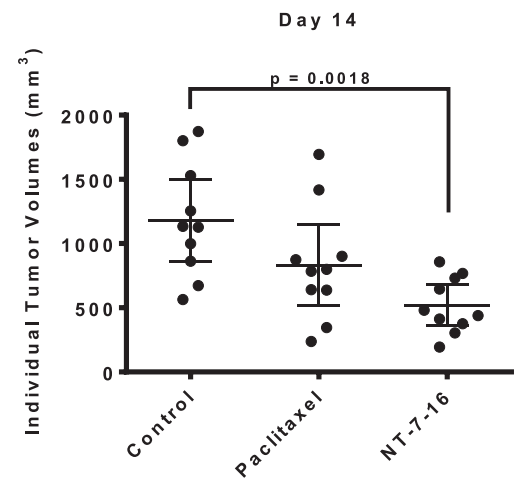

C

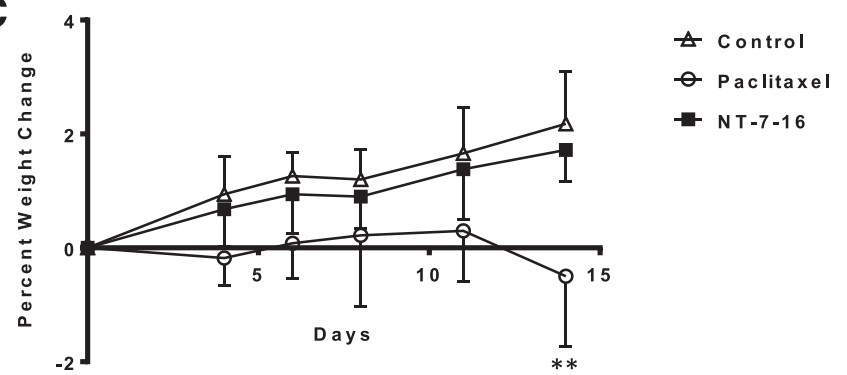

Fig. 5. Antitumor effects of NT-7-16. (A) The effects of NT-7-16 on MDAMB-435 tumors were evaluated in nude mice. The graph shows the average tumor volume \pm S.D. starting on day 0 . After the tumors were established, mice were treated daily by i.p. injection of $75 \mathrm{mg} / \mathrm{kg}$ NT-7-16. Paclitaxel was dosed at $20 \mathrm{mg} / \mathrm{kg}$ twice weekly. (B) Individual tumor volumes on day 14 are presented; the mean is represented by the horizontal line in each panel $\pm 95 \%$ confidence intervals. Statistical analysis was performed using a one-way ANOVA with a Tukey's post hoc test to compare each condition with every other condition. A significant difference $(P=0.0018)$ was only found between the control and NT-7-16 groups. (C) Average percent weight change for each treatment group over the course of the trial \pm S.D. Statistical analysis was performed using a one-way ANOVA with a Dunnet's post hoc test to compare each drug treated condition to the control. A significant difference $(P=0.0014)$ was only found between the control and NT-7-16 groups.

contains Cys241 $\beta$, which is the site predicted to bind our optimized pyrroles. In the current study, we focused on identifying compounds with optimal binding into this subpocket, but have not evaluated how the refined model predicts interactions with compounds that would occupy the other sites and sub-pockets within the colchicine domain.

The major drawbacks of the pyrrole-based compounds are their limited aqueous solubility and low in vivo potency. The parent compound JG-03-14 had poor aqueous solubility, which necessitated use of DMSO as the solvent for in vivo studies. The aqueous solubility of NT-7-16 has been improved from JG03-14 and allowed the use of a $70 \%$ aqueous vehicle, but is still not optimal. Even though excellent antitumor effects were observed with daily administration of $75 \mathrm{mg} / \mathrm{kg}$, an MTD was never reached because of its limited solubility. Multiple colchicine site agents with excellent aqueous solubility, in vivo potency and metabolic stability have been identified (Gangjee et al., 2013; Lu et al., 2014; Zhang et al., 2014) so it is reasonable that newer pyrrole analogs with improved aqueous solubility can be generated. Efforts will also be made to increase the in vivo potency. The metabolic liabilities of the trimethoxyphenyl moieties were identified in other colchicine site binding agents ( $\mathrm{Li}$ et al., 2011) and medicinal chemistry optimization has improved these liabilities while still retaining potent cytotoxic activities (Lu et al., 2014). Identification of analogs with better aqueous solubility and in vivo potency will be a future priority.

In spite of these limitations, an advantage of NT-7-16 is its low toxicity. In the in vivo trial, NT-7-16 had excellent antitumor effects with no weight loss or other side effects, suggesting that it is less toxic than CA-4, which has an MTD of $500-1,000 \mathrm{mg} / \mathrm{kg}$ in a variety of xenograft models (Dark et al., 1997; Horsman et al., 1998; Grosios et al., 1999; Nabha et al., 2001; Chaplin and Hill, 2002). We hypothesize that the optimal fit of NT-7-16 deep within the $\beta$-tubulin pocket that allows interactions with both Cys241 $\beta$ and Ala354 $\beta$ might reduce toxicity. This is consistent with clinical trials results of T138067, which also is buried deep in $\beta$-tubulin and binds covalently with Cys241 $\beta$ and had no dose limiting toxicities or neurotoxicity (Kirby et al., 2005; Berlin et al., 2008). The low toxicity of NT-7-16 is also reminiscent of 2-methoxyestradiol, a drug that was advanced into the clinic due to its antiangiogenic activities. A clinical trial of 2-methoxyestradiol showed that it was safe and a maximal tolerated dose was not achieved (James et al., 2007); unfortunately it did not have the efficacy necessary to advance to Phase III trials.

A number of other colchicine site agents that rapidly disrupt tumor vasculature have entered clinical evaluation. The failure of these vascular disrupting agent drugs to advance clinically, due in part to toxicity, brings up a question of whether different poses and interactions within the colchicine site might have different effects on tumor vasculature. Recent data suggests that vascular normalization might be a more optimal therapeutic strategy than vascular disruption (Rivera and Bergers, 2015) and thus colchicine site agents without vascular disrupting action might have advantages in clinical trials. When we identify a clinical lead candidate it will be valuable to conduct functional MRI studies to evaluate tumor perfusion.

In summary, these studies demonstrate that modeling of pyrrole compounds within the refined colchicine site can identify optimized colchicine site agents with excellent in vitro and in vivo activities. Further optimization of their medicinal chemical properties will now be required to translate this improved fit within the site into greater potency and efficacy both in vitro and in vivo. We can also not rule out potential metabolic and pharmacokinetic liabilities that will need to be optimized as this class of pyrrole compounds progresses through further lead optimization and preclinical studies.

\section{Acknowledgments}

The authors thank Dr. Diane Kellogg of the University of Richmond for analytical support on this project. 


\section{Authorship Contributions}

Participated in research design: Rohena, Risinger, Mooberry, Gupton, Telang, Sikorski.

Conducted experiments: Rohena, Risinger, Da, Telang.

Contributed new reagents or analytic tools: Telang.

Performed data analysis: Rohena, Risinger, Mooberry, Da, Kellogg,

Gupton.

Wrote or contributed to the writing of the manuscript: Rohena, Risinger, Kellogg, Gupton, Sikorski, Mooberry, Telang.

\section{References}

Bennett MJ, Barakat K, Huzil JT, Tuszynski J, and Schriemer DC (2010) Discovery and characterization of the laulimalide-microtubule binding mode by mass shift perturbation mapping. Chem Biol 17:725-734.

Berlin JD, Venook A, Bergsland E, Rothenberg M, Lockhart AC, and Rosen L (2008) Phase II trial of T138067, a novel microtubule inhibitor, in patients with metastatic, refractory colorectal carcinoma. Clin Colorectal Cancer 7:44-47.

Bhattacharyya B and Wolff J (1974) Promotion of fluorescence upon binding of colchicine to tubulin. Proc Natl Acad Sci USA 71:2627-2631.

Burnett JC, Botti P, Abraham DJ, and Kellogg GE (2001) Computationally accessible method for estimating free energy changes resulting from site-specific mutations of biomolecules: systematic model building and structural/hydropathic analysis of deoxy and oxy hemoglobins. Proteins 42:355-377.

Chakraborti S, Chakravarty D, Gupta S, Chatterji BP, Dhar G, Poddar A, Panda D, Chakrabarti P, Ghosh Dastidar S, and Bhattacharyya B (2012) Discrimination of ligands with different flexibilities resulting from the plasticity of the binding site in tubulin. Biochemistry 51:7138-7148.

Chaplin DJ and Hill SA (2002) The development of combretastatin A4 phosphate as a vascular targeting agent. Int $J$ Radiat Oncol Biol Phys 54:1491-1496.

Cozzini P, Fornabaio M, Marabotti A, Abraham DJ, Kellogg GE, and Mozzarelli A (2004) Free energy of ligand binding to protein: evaluation of the contribution of water molecules by computational methods. Curr Med Chem 11:3093-3118.

Da C, Mooberry SL, Gupton JT, and Kellogg GE (2013a) How to deal with lowresolution target structures: using SAR, ensemble docking, hydropathic analysis, and 3D-QSAR to definitively map the $\alpha \beta$-tubulin colchicine site. J Med Chem $\mathbf{5 6}$ $7382-7395$

Da C, Telang N, Barelli P, Jia X, Gupton JT, Mooberry SL, and Kellogg GE (2012) Pyrrole-based antitubulin agents: two distinct binding modalities are predicted for C-2 analogs in the colchicine site. ACS Med Chem Lett 3:53-57.

Da C, Telang N, Hall K, Kluball E, Barelli P, Finzel K, Jia X, Gupton JT, Mooberry SL, and Kellogg GE (2013b) Developing novel C-4 analogues of pyrrole-based antitubulin agents: weak but critical hydrogen bonding in the colchicine site. MedChemComm 4:417-421.

Dark GG, Hill SA, Prise VE, Tozer GM, Pettit GR, and Chaplin DJ (1997) Combretastatin A-4, an agent that displays potent and selective toxicity toward tumor vasculature. Cancer Res 57:1829-1834.

Dorléans A, Gigant B, Ravelli RB, Mailliet P, Mikol V, and Knossow M (2009) Variations in the colchicine-binding domain provide insight into the structural switch of tubulin. Proc Natl Acad Sci USA 106:13775-13779.

Dumontet C and Jordan MA (2010) Microtubule-binding agents: a dynamic field of cancer therapeutics. Nat Rev Drug Discov 9:790-803.

Gangjee A, Zhao Y, Lin L, Raghavan S, Roberts EG, Risinger AL, Hamel E, and Mooberry SL (2010) Synthesis and discovery of water-soluble microtubule targeting agents that bind to the colchicine site on tubulin and circumvent Pgp mediated resistance. $J$ Med Chem 53:8116-8128.

Gangjee A, Zhao Y, Raghavan S, Rohena CC, Mooberry SL, and Hamel E (2013) Structure-activity relationship and in vitro and in vivo evaluation of the potent cytotoxic anti-microtubule agent $\mathrm{N}$-(4-methoxyphenyl)-N,2,6-trimethyl-6,7dihydro-5H-cyclopenta[d]pyrimidin-4-aminium chloride and its analogues as antitumor agents. J Med Chem 56:6829-6844.

Gigant B, Wang C, Ravelli RB, Roussi F, Steinmetz MO, Curmi PA, Sobel A, and Knossow M (2005) Structural basis for the regulation of tubulin by vinblastine. Nature 435:519-522.

Grosios K, Holwell SE, McGown AT, Pettit GR, and Bibby MC (1999) In vivo and in vitro evaluation of combretastatin A-4 and its sodium phosphate prodrug. $\mathrm{Br} J$ Cancer 81:1318-1327.

Gupton JT, Burham BS, Krumpe K, Du K, Sikorski JA, Warren AE, Barnes CR, and Hall IH (2000) Synthesis and cytotoxicity of 2,4-disubstituted and 2,3,4trisubstituted brominated pyrroles in murine and human cultured tumor cells. Arch Pharm (Weinheim) 333:3-9.

Hamel E (2002) Interactions of antimitotic peptides and depsipeptides with tubulin. Biopolymers 66:142-160.

Hamel E (2003) Evaluation of antimitotic agents by quantitative comparisons of their effects on the polymerization of purified tubulin. Cell Biochem Biophys 38:1-22.

Horsman MR, Ehrnrooth E, Ladekarl M, and Overgaard J (1998) The effect of combretastatin A-4 disodium phosphate in a $\mathrm{C} 3 \mathrm{H}$ mouse mammary carcinoma and a variety of murine spontaneous tumors. Int J Radiat Oncol Biol Phys 42:895-898.
Huzil JT, Chik JK, Slysz GW, Freedman H, Tuszynski J, Taylor RE, Sackett DL, and Schriemer DC (2008) A unique mode of microtubule stabilization induced by peloruside A. J Mol Biol 378:1016-1030.

James J, Murry DJ, Treston AM, Storniolo AM, Sledge GW, Sidor C, and Miller KD (2007) Phase I safety, pharmacokinetic and pharmacodynamic studies of 2-methoxyestradiol alone or in combination with docetaxel in patients with locally recurrent or metastatic breast cancer. Invest New Drugs 25:41-48.

Joe PA, Banerjee A, and Ludueña RF (2008) The roles of cys124 and ser239 in the functional properties of human betaIII tubulin. Cell Motil Cytoskeleton 65:476-486

Jones G, Willett P, and Glen RC (1995) Molecular recognition of receptor sites using a genetic algorithm with a description of desolvation. J Mol Biol 245:43-53.

Jordan MA and Wilson L (2004) Microtubules as a target for anticancer drugs. Nat Rev Cancer 4:253-265.

Kellogg EG and Abraham DJ (2000) Hydrophobicity: is $\log \mathrm{P}(\mathrm{o} / \mathrm{w})$ more than the sum of its parts? Eur J Med Chem 35:651-661.

Kirby S, Gertler SZ, Mason W, Watling C, Forsyth P, Aniagolu J, Stagg R, Wright M, Powers J, and Eisenhauer EA (2005) Phase 2 study of T138067-sodium in patients with malignant glioma: trial of the National Cancer Institute of Canada Clinical Trials Group. Neuro-oncol 7:183-188.

Lee L, Robb LM, Lee M, Davis R, Mackay H, Chavda S, Babu B, O'Brien EL, Risinger $\mathrm{AL}$, and Mooberry SL et al. (2010) Design, synthesis, and biological evaluations of 2,5-diaryl-2,3-dihydro-1,3,4-oxadiazoline analogs of combretastatin-A4. J Med Chem 53:325-334.

Li CM, Wang Z, Lu Y, Ahn S, Narayanan R, Kearbey JD, Parke DN, Li W, Miller DD, and Dalton JT (2011) Biological activity of 4-substituted methoxybenzoyl-arylthiazole: an active microtubule inhibitor. Cancer Res 71:216-224.

Lu Y, Chen J, Wang J, Li CM, Ahn S, Barrett CM, Dalton JT, Li W, and Miller DD (2014) Design, synthesis, and biological evaluation of stable colchicine binding site tubulin inhibitors as potential anticancer agents. $J$ Med Chem 57:7355-7366.

$\mathrm{Lu}$ Y, Chen J, Xiao M, Li W, and Miller DD (2012) An overview of tubulin inhibitors that interact with the colchicine binding site. Pharm Res 29:2943-2971.

Mooberry SL, Weiderhold KN, Dakshanamurthy S, Hamel E, Banner EJ, Kharlamova A, Hempel J, Gupton JT, and Brown ML (2007) Identification and characterization of a new tubulin-binding tetrasubstituted brominated pyrrole. Mol Pharmacol 72:132-140.

Nabha SM, Mohammad RM, Wall NR, Dutcher JA, Salkini BM, Pettit GR, and AlKatib AM (2001) Evaluation of combretastatin A-4 prodrug in a non-Hodgkin's lymphoma xenograft model: preclinical efficacy. Anticancer Drugs 12:57-63.

Nogales E, Wolf SG, Khan IA, Ludueña RF, and Downing KH (1995) Structure of tubulin at 6.5 A and location of the taxol-binding site. Nature 375:424-427.

Prota AE, Bargsten K, Diaz JF, Marsh M, Cuevas C, Liniger M, Neuhaus C, Andreu JM, Altmann KH, and Steinmetz MO (2014) A new tubulin-binding site and pharmacophore for microtubule-destabilizing anticancer drugs. Proc Natl Acad Sci USA 111:13817-13821.

Ravelli RB, Gigant B, Curmi PA, Jourdain I, Lachkar S, Sobel A, and Knossow M (2004) Insight into tubulin regulation from a complex with colchicine and a stathmin-like domain. Nature 428:198-202.

Risinger AL, Jackson EM, Polin LA, Helms GL, LeBoeuf DA, Joe PA, Hopper-Borge E, Ludueña RF, Kruh GD, and Mooberry SL (2008) The taccalonolides: microtubule stabilizers that circumvent clinically relevant taxane resistance mechanisms. Cancer Res 68:8881-8888.

Rivera LB and Bergers G (2015) CANCER: tumor angiogenesis, from foe to friend. Science 349:694-695.

Sarkar A and Kellogg GE (2010) Hydrophobicity: shake flasks, protein folding and drug discovery. Curr Top Med Chem 10:67-83.

Spyrakis F, Amadasi A, Fornabaio M, Abraham DJ, Mozzarelli A, Kellogg GE, and Cozzini P (2007) The consequences of scoring docked ligand conformations using free energy correlations. Eur J Med Chem 42:921-933.

Tripathi A, Fornabaio M, Kellogg GE, Gupton JT, Gewirtz DA, Yeudall WA, Vega NE, and Mooberry SL (2008) Docking and hydropathic scoring of polysubstituted pyrrole compounds with antitubulin activity. Bioorg Med Chem 16:2235-2242.

Verma S, Miles D, Gianni L, Krop IE, Welslau M, Baselga J, Pegram M, Oh DY, Diéras V, and Guardino E et al.; EMILIA Study Group (2012) Trastuzumab emtansine for HER2-positive advanced breast cancer. $N$ Engl $J$ Med 367: 1783-1791

Xiao H, Verdier-Pinard P, Fernandez-Fuentes N, Burd B, Angeletti R, Fiser A, Horwitz SB, and Orr GA (2006) Insights into the mechanism of microtubule stabilization by Taxol. Proc Natl Acad Sci USA 103:10166-10173.

Zhang X, Raghavan S, Ihnat M, Thorpe JE, Disch BC, Bastian A, Bailey-Downs LC, Dybdal-Hargreaves NF, Rohena CC, and Hamel E et al. (2014) The design and discovery of water soluble 4-substituted-2,6-dimethylfuro[2,3-d]pyrimidines as multitargeted receptor tyrosine kinase inhibitors and microtubule targeting antitumor agents. Bioorg Med Chem 22:3753-3772.

Address correspondence to: Susan L. Mooberry, Department of Pharmacology, Mail Code 7764, 7703 Floyd Curl Drive, University of Texas Health Science Center at San Antonio, San Antonio, TX, 78229-3900. E-mail: Mooberry@uthscsa.edu. 